\title{
Uso del mapa conceptual como estrategia didáctica para mejorar la comprensión lectora
}

\section{Using the conceptual map as a teaching strategy to improve reading comprehension}

\author{
Cecilia Toro Soler * \\ Consuelo Esperanza Cepeda Cepeda ** \\ Luz Esperanza Gil Sánchez
}

Fecha de envío: 23 de mayo de 2017

Fecha de aprobación: 3 de noviembre de 2017

Artículo de investigación científica y tecnológica

\section{Resumen}

Este artículo tiene como objetivo describir el contexto educativo de la comprensión lectora de los estudiantes de $3^{\circ}$ y $4^{\circ}$ grado de la I. E. Sergio Camargo (Municipio de Miraflores). Se pretende demostrar el efecto que existe entre el uso de los mapas conceptuales y el mejoramiento en la comprensión lectora. Mediante la metodología cualitativa, con un enfoque crítico social y un diseño basado en la investigación acción, se puede encontrar que el primer personaje pedagógico que estimula a los estudiantes en emular ideas y formas de la comprensión lectora, es el docente, dando ejemplos y organizando los ejercicios, de tal modo que si este agente principal no encuentra estrategias adecuadas para mejorar el proceso de comprensión lectora sino que lo hace de una manera monótona, implícitamente hace de la lectura y la comprensión lectora un deber y una obligación para los niños, leer de este modo genera una disminución en el interés propio y autónomo por parte
*Institución Educativa Sergio Camargo_, Boyacá-Colombia cetoso07@hotmail.com **Institución Educativa Sergio Camargo_, Boyacá-Colombia consuelo3cepeda@gmail. com

*** Institución Educativa Sergio Camargo_, Boyacá-Colombia luzespe1975@gmail.com

(c) (1) (3) 
de los estudiantes, además que dificulta en los educandos la posibilidad de que ellos plasmen sus propias ideas. En este sentido, la utilización de los mapas conceptuales como estrategia influye en la comprensión lectora y ayuda a

\section{Abstract}

This article aims to describe the educational context of reading comprehension of students in 3rd and 4th grade of the educational institution "Sergio Camargo" (Miraflores, Colombia). $\mathrm{t}$ is intended to demonstrate the effect between the use of conceptual maps and the improvement in the reading comprehension by means of the qualitative methodology, with a critical social approach and a design based on the research-action, it can be found that the first pedagogical character that stimulates students to emulate ideas and forms of reading comprehension, is the teacher, giving examples and organizing the exercises, in such a way that if this principal agent does not find adequate mejorar en el rendimiento académico de manera positiva, pronosticando el éxito de los estudiantes.

Palabras clave: mapa conceptual, estrategia didáctica, comprensión lectora, aprendizaje significativo. strategies to improve the process of reading comprehension and he does it in a monotonous way, implicitly makes reading and reading comprehension a duty and an obligation for children. Reading in this way generates a decrease in self-interest on the students, also making it difficult for learners the chance for them to capture their own ideas. In this sense, the use of concept maps as a strategy influences reading comprehension and helps improve academic performance in a positive way, predicting the success of students.

Keywords: conceptual map, didactic strategy, reading comprehension, meaningful learning. 


\section{Introducción}

La lectura es una herramienta básica que facilita y posibilita los demás aprendizajes, por lo tanto se convierte en una actividad esencial para la adquisición de nuevos conocimientos. La lectura es la base para la enseñanza, por esa razón debemos preocuparnos por la formación de buenos lectores.

La comprensión lectora, es uno de los elementos indispensables para el aprendizaje de los educandos, tanto que en suma con la lectoescritura, casi que determinan el éxito o el fracaso de los niños en el contexto escolar. La comprensión lectora hace referencia a un proceso psicológico y pedagógico relevante en el aprendizaje de conocimientos, que, por su carácter transversal, permite a los educandos conocer y hacer relaciones con los contenidos temáticos. La comprensión lectora es un instrumento de vital importancia en todas las esferas de la vida de los niños y los sujetos, es un proceso que sigue estando vigente a pesar de la aparición de nuevas vías y medios de asimilación de conocimientos: la lectura continúa siendo uno de los ejes fundamentales para apropiar la información del mundo que rodea al ser humano.

Existen docentes que buscan mejorar el proceso de comprensión lectora mediante el uso de diferentes estrategias, pero vale agregar que la preocupación y las acciones que se desarrollan no son suficientes, debido a que las propuestas que se desarrollan se piensan más desde la preocupación sobre cómo enseñar unos temas, que desde la articulación, las expectativas y gustos de los estudiantes. Con las fortalezas de los docentes, las orientaciones de la Institución Educativa y las políticas educativas del orden nacional. "En consecuencia, el acto educativo se ha tornado en instrumento de normalización que, en ocasiones, tienden a homogenizar las distintas dinámicas y fuerzas que, espontáneamente, surgen como parte del proceso creador que debe tener núcleo en el aula y la escuela."(Cañizalez \& Benavides, 2016, p. 109) el problema se encuentra en asumir la formación de los niños y jóvenes cómo un proceso estandarizado y no como un acto cultural, político y ético que requiere ser trabajado no sólo desde la razón, sino también desde la emoción.

A pesar de todas estas problemáticas entorno a la comprensión lectora de los educandos, se han hecho esfuerzos políticos para mejorar esta situación. En el marco del programa de Calidad para la Equidad, del Ministerio de Educación Nacional, se desarrolla desde el 2010, el Plan Nacional de Lectura y Escritura (PNLE), que busca garantizar el acceso a la cultura desde la lectura, como vía de equidad e inclusión social y de desarrollo de la ciudadanía. Por el cual es una iniciativa del gobierno nacional, liderada por los Ministerios de Educación y de Cultura, para que los sectores público, privado, solidario y la sociedad civil del país se unan entorno a un objetivo común: lograr que los colombianos incorporen la lectura a su vida cotidiana, que los niños, niñas y jóvenes, lean y escriban de una mejor forma, y lo disfruten. Además, fomenta el desarrollo de las
La comprensión lectora, es uno de los elementos indispensables para el aprendizaje de los educandos, tanto que en suma con la lectoescritura, casi que determinan el éxito o el fracaso de los niños en el contexto escolar. 
Los resultados en pruebas saber año 2016, en el área de español, arrojan lo siguiente: insuficiente, el 33\%; mínimo, el $13 \%$; satisfactorio, el 33\%; y avanzado, el $21 \%$. competencias comunicativas, a través del mejoramiento del comportamiento lector, la comprensión lectora y la producción textual de estudiantes de educación preescolar, básica y media, fortaleciendo la escuela como espacio fundamental para la formación de lectores y escritores, y vinculando la familia en estos procesos (MEN, 2013).

Con respecto a lo anterior, en el departamento de Boyacá se adelantan acciones dentro del Plan Nacional de Lectura y Escritura a través de la secretaría de Cultura y Turismo de Boyacá, La Red de Bibliotecas Públicas y la Corporación Estanislao Zuleta, adelantando talleres dirigidos a la población de primera infancia, jóvenes y adultos mayores, permitiendo así un mayor acceso y participación a toda la ciudadanía al incentivar el gusto por la lectura y escritura desde los primeros años.

Todas estas situaciones anteriormente mencionadas, en las regiones e instituciones educativas del Departamento de Boyacá, no están exentas en el Municipio de Miraflores. La Institución Educativa Sergio Camargo, presta sus servicios en el área rural y urbana en los niveles de preescolar, básica, media: académica y técnica con especialidades en comercio y sistemas. Cuenta con una población cerca de 1.100 estudiantes atendidos por un equipo de 48 docentes, 9 administrativos, 4 directivos docentes y la psicoorientadora escolar. Se ofrecen programas de educación no formal para jóvenes y adultos como CEDEBOY y Telesecundaria.
Actualmente, la Institución Educativa se encamina en un proceso de mejoramiento constante que tiene como meta elevar su nivel académico en pruebas SABER tercero, quinto, noveno y once, supérate en el saber, simulacros saber ICFES, entre otras.

Sin embargo, el problema en parte radica en la manera de proceder de los estudiantes para alcanzar la comprensión de un texto, debido a que saben lo que deben lograr, es decir, la comprensión; pero, desconocen cómo llegar a ella. Lo anterior se hace latente en el aula de clase cuando se les cuestiona sobre lo comprendido y sólo se escucha el silencio de sus voces y rostros pensativos, luego, algunos se devuelven a la lectura respondiendo con los mismos párrafos de ésta; no hacen construcciones propias, ni concatenan los nuevos conocimientos con los previos.

Todas estas situaciones terminan afectando la manera y elementos que utilizan los educandos para relacionarse con los otros y el medio, y sobre todo con los resultados en las pruebas saber.

Los resultados en pruebas saber año 2016, en el área de español, arrojan lo siguiente: insuficiente, el 33\%; mínimo, el $13 \%$; satisfactorio, el $33 \%$; y avanzado, el $21 \%$. Con esto, se quiere decir que el nivel en lenguaje es mínimo, puesto que al estudiante promedio de este nivel, ante textos narrativos e informativos cortos, de estructura sencilla y con contenidos cotidianos, se le dificulta: buscar información dentro del texto a partir de marcas textuales evidentes o fácilmente identificables; establecer 
relaciones temporales entre eventos del texto cuando están ordenados dentro de la misma linealidad del escrito; dificultad en la recuperación de la información explícita y local, dificultad en la identificación de la intención comunicativa en actos de habla simples y de uso cotidiano.(Resultados ICFES saber tercero, quinto y noveno, 2016)

A causa de estos resultados, se observa que los componentes evaluados en lenguaje, como semántico, sintáctico y pragmático, son las debilidades que se deben fortalecer para favorecer la comprensión lectora de los estudiantes. Hay que mencionar, además, que se observa que los alumnos siguen estudiando para una prueba, que pasan al siguiente grado y olvidan conocimientos adquiridos en el grado anterior, que tienen dificultades de concentración, muestran intereses ajenos a la parte académica. Los niños y niñas de los grados terceros y cuarto de la Institución Educativa Sergio Camargo, presentan bajos resultados en su rendimiento académico, lo cual ha tenido implicaciones en los desempeños que ellos han mostrado en las pruebas SABER. El análisis de las posibles causas de esta situación problémica, ha permitido determinar que las deficiencias en comprensión lectora repercuten en los logros académicos, tanto de lengua castellana, como de otras áreas, toda vez que los estudiantes tienen dificultad para dar cuenta del sentido de los textos que leen, por la limitación para conectar ideas entre sí y para establecer jerarquías y relaciones entre ellas como lo demuestra el índice sintético. La situación problémica descrita anteriormente, ha motivado el diseño e implementación de variadas acciones y estrategias, tales como: comunidades de indagación, preguntas problematizadoras, fichas temáticas, fichas analíticas, simulacros de pruebas, proyectos de aula, Proyecto Institucional de Lectura y Oratoria (PILO); estrategias que no han tenido el efecto esperado sobre la mejora de los educandos mediante la comprensión lectora, pero se continúa en el proceso.

La lectura es una tarea que necesita de ciertas herramientas, que favorezcan las habilidades cuando de entender, comprender y producir se trata. Estanislao Zuleta (1982) decía: "no hay textos fáciles, ni textos difíciles de leer, lo que hay son lectores fáciles y difíciles". Se vive en una sociedad donde se margina, se prohíbe, se censura y, en ocasiones, hasta se manipula a los lectores en cuanto a qué leer, qué no leer, olvidando que existen diversidad de gustos y apreciaciones, descartando la importancia del verdadero beneficio de saber leer. Por estas razones y muchas otras, es indispensable superar el bloqueo epistemológico, axiológico y psicológico, que permita libertad de expresión en la sociedad, la cultura y en la misma educación. La lectura forma verdaderos hablantes, escuchas, lectores y escritores creativos y críticos. En lo pedagógico, a su vez, hay que hacer un trabajo en muchas direcciones: leer cuentos, prensa, los magazines, libros en diferentes ciencias y artes, sin descuidar otros medios masivos como la televisión o la radio, pero con sentido crítico, con la reflexión y el cambio de opiniones.
La lectura es una tarea que necesita de ciertas herramientas, que favorezcan las habilidades cuando de entender, comprender $\mathrm{y}$ producir se trata. 


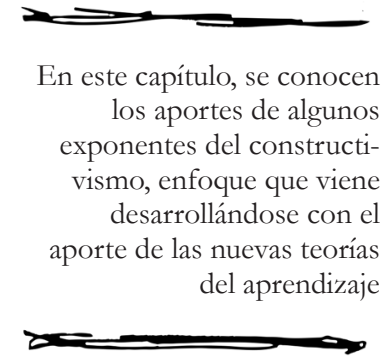

Debemos apoyarnos en propuestas didácticas basadas en organizadores gráficos que reporten sentido a los textos que se leen. Estos organizadores se fundamentan en teorías del aprendizaje, como: Teoría de la Asimilación de David Ausubel (1963); Teoría del procesamiento de información de Jorge Miller (1962), que permite o da la oportunidad de crear diseños de organizadores gráficos, tales como: mapas conceptuales, diagrama jerárquico, cadena de secuencias, rueda de atributos, esquemas, mapas de ideas, telarañas, entre otros, que se adecuen al nivel de los niños y al tipo de texto que van a usar. La Institución Educativa y más exactamente el salón de clase, es un lugar apto para "escuchar" a los niños, conocer sus intereses, necesidades, sentimientos e inquietudes; los aprendizajes están inmersos en la cotidianidad de los niños, esto es lo que hace que tengan sentido y significado para ellos. Vygotsky (1991) se basa principalmente en el aprendizaje sociocultural de cada individuo y, por lo tanto, en el medio en el cual se desarrolla.

Se pretende mejorar y generar unos ambientes de aprendizaje más atractivos y felices para los estudiantes y para los docentes, es decir, fomentar el gusto por aprender y por mejorar la comprensión lectora, por otra parte, hacer uso de herramientas del contexto desde la misma naturaleza hasta los avances tecnológicos; buscando comprometer en el proceso a todos los demás compañeros.

De esta manera, se logra transformar y dinamizar las prácticas pedagógicas; vale la pena decir, que los beneficios esperados serían en cuatro aspectos, primero desde los ambientes escolares, puesto que debe haber un cambio actitudinal de los estudiantes y docentes en el proceso de enseñanza aprendizaje; segundo, el pedagógico, puesto que el aprendizaje se vuelve significativo; tercero, lo social, porque le permite al estudiante con este aprendizaje recrear y transformar su realidad; y cuarto, en la evaluación, porque se mejoran las pruebas externas e internas de la Institución Educativa.

\section{Marco teórico}

En este capítulo, se conocen los aportes de algunos exponentes del constructivismo, enfoque que viene desarrollándose con el aporte de las nuevas teorías del aprendizaje: Lev Vygotsky (1987,1988), David Ausubel (1963,1968), y Novak y Gowin (1977, 1988).

Actualmente, algunas palabras de uso son: aprendizaje significativo, cambio conceptual y constructivismo. Una buena enseñanza debe ser constructivista, promover el cambio conceptual y facilitar el aprendizaje significativo.

Es probable que la práctica docente aún tenga mucho del conductismo, pero el discurso es cognitivista/ constructivista/significativo. Lo que se quiere decir es que puede que no haya, aún, un verdadero cambio conceptual en este sentido, pero parece que se está caminando en esa dirección.

\section{Mapa conceptual}

Es un organizador gráfico que revela la forma en que se relacionan los conceptos entre sí. Va de lo general a lo particular y se lee de arriba hacia abajo. 
Son muy importantes los conectores, ya que le dan sentido a la lectura.

Los mapas conceptuales tienen como objetivo dirigir la atención hacia aquellas ideas o nociones más relevantes dentro de un documento específico. Novak y Gowin (1998) manifiestan que los mapas conceptuales favorecen la adquisición de aprendizajes significativos, puesto que permiten establecer relaciones conceptuales yjerárquicas entre diferentes significados, exigiendo al alumno que interiorice los nuevos conceptos que le son presentados englobándolos bajo otros más amplios e inclusivos. De este modo, los mapas conceptuales establecen conexiones complejas entre diferentes conceptos, que unidos a través de proposiciones constituyen una unidad semántica que proporciona un resumen gráfico de todo lo que se ha interiorizado y aprendido a través de su realización. Así, con esta actividad, los alumnos pueden representar mediante dos o más jerarquías un conjunto de significados conceptuales incluidos en una estructura de proposiciones.

Los mapas conceptuales se enmarcan dentro del enfoque constructivista, el cual presenta principios epistemológicos, tales como:

\section{Estrategias de aprendizaje}

Las estrategias de aprendizaje son procedimientos (conjuntos de pasos, operaciones o habilidades) que un aprendiz emplea en forma consciente, controlada e intencional, como instrumentos flexibles para aprender significativamente y solucionar problemas (Díaz Barriga, Castañeda y Lule, 1986).

\section{Concepto de la lectura:}

¿Qué es leer?

Leer es un proceso de interacción entre el lector y el texto, proceso mediante el cual el primero intenta satisfacer (obtener una información pertinente para) los objetivos que guían su lectura.

\section{La comprensión lectora}

La comprensión lectora es la habilidad que posee un individuo de interpretar un texto, a partir de la reflexión y el análisis. Según (Núñez, 2009), comprender el lenguaje es un proceso complejo integrado por multitud de subprocesos más sencillos que, en muchos aspectos, difieren notablemente unos de otros. La comprensión del mensaje comienza con la percepción del lenguaje, pasando por la comprensión del significado general de la enunciación hasta culminar en la comprensión de aspectos parciales; es decir, el receptor nunca se propone comprender palabras o frases aisladas, sino captar el significado global de toda la comunicación, su sentido interno. "Al hablar de lectura no se habla necesariamente de lo textual, sino también de la lectura audiovisual, lectura del arte, lectura de los acontecimientos, lectura del mundo, etc.'(Mariño, Pulido \& Morales, 2016, p. 97)

\section{Metodología}

El marco metodológico presenta los elementos que permiten guiar el desarrollo del proceso de investigación en cada uno de los momentos, es decir, se plantean los métodos y las técnicas, los instrumentos y la forma con la
Los mapas conceptuales tienen como objetivo dirigir la atención hacia aquellas ideas o nociones más relevantes dentro de un documento específico. 


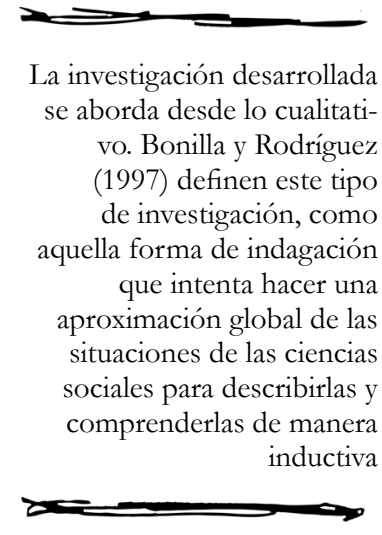

que se va a abordar el desarrollo de la investigación; para lo cual se hace necesario establecer un campo de acción, en otras palabras, la población y la muestra.

\section{Enfoque y tipo de investigación}

La investigación desarrollada se aborda desde lo cualitativo. Bonilla y Rodríguez (1997) definen este tipo de investigación, como aquella forma de indagación que intenta hacer una aproximación global de las situaciones de las ciencias sociales para describirlas y comprenderlas de manera inductiva, es decir, a partir de los conocimientos, experiencias, creencias y representaciones que tienen las diferentes personas involucradas en ellas. Esto supone que los individuos interactúan con los otros miembros de su contexto social compartiendo el significado y el conocimiento que tienen de sí mismos y la realidad.

El enfoque asumido para este proyecto de investigación, es el Crítico-social, definido como la perspectiva que sirve al interés emancipatorio, hacia la libertad y la autonomía racional, procurando ofrecer a los individuos un medio para concientizarse de cómo sus objetivos y propósitos pueden haber resultado distorsionados o reprimidos y especificar cómo erradicarlos, de manera que posibilite la búsqueda de las verdaderas metas.

Sumado a lo anterior, el diseño escogido es la IA. Este diseño implica poner en permanente práctica la teoría, desde un diálogo entre saberes teóricos y saberes prácticos, convirtiendo al investigador en un educador activo que, en este caso, busca mejorar el proceso de comprensión lectora mediante el uso del mapa conceptual. Desde acá se desarrolla y comprende "el pausado ritmo de reflexión y acción" que debe acompañar los procesos de investigación en un camino en "espiral", haciendo de esta modalidad de investigación el fundamento de una ciencia social crítica (Borda, 2004; Kemmis, 1992).

\section{Población y muestra}

La propuesta se desarrolla en el municipio de Miraflores, Institución Educativa Sergio Camargo, sedes Rafael Uribe y El Bosque, ubicadas en la zona urbana. Estas sedes atienden a una población de 396 estudiantes en total. Como muestra, se toman el grado tercero y un curso cuarto, y se trabaja con 98 estudiantes de género masculino y femenino, cuyas edades están entre los 8 y 12 años, procedentes de estratos socioeconómicos 1 y 2 , cuya mayoría de familias de procedencia están conformados por papá, mamá y hermanos; todos ellos registrados en el SIMAT (Sistema integrado de matrícula) en el año lectivo 2017.

\section{Técnicas e instrumentos de recolección de la información cualitativa}

\section{Técnicas de recolección de datos}

Observación Participante. Sandoval (2002) define esta técnica como aquella que surge como una alternativa distinta a las formas de observación convencional. El estudio de los problemas depende de la forma en que las preguntas sean dirigidas, 
refinadas, elaboradas y focalizadas a través del proceso de recolección de datos.

Taller investigativo. Su fortaleza principal estriba en la posibilidad que brinda el abordar, desde una perspectiva integral y participativa, situaciones sociales que requieren algún tipo de análisis. Esto significa que el taller no es solo una estrategia de recolección de información, sino también, de análisis y de planeación. La operatividad y eficacia de esta estrategia requiere un alto compromiso de los actores y una gran capacidad de convocatoria animación, y conducción de los investigadores.

Los relatos de vida. "Los relatos de vida ofrecen un marco interpretativo a través del cual el sentido de la experiencia humana se revela en relatos personales en un modo que da prioridad a las explicaciones individuales de las acciones más que a los métodos que filtran y ordenan las respuestas en categorías conceptuales predeterminadas" (Torres, 1999, p.37). Se busca un relato puramente subjetivo, adoptando un planteamiento de interpretar significados, más que de encontrar causalidades.

La entrevista semiestructurada. Se determina cuál es la información relevante que se desea conseguir, realizando preguntas abiertas en las asesorías individuales acerca de las dificultades y motivos generadores de bajo rendimiento académico (Sandoval, 2002).

\section{Instrumentos de recolección de la información}

Enla parteinvestigativa de características cualitativas, se utilizan los siguientes instrumentos de recolección de datos:
Diario de campo. Su objeto es registrar la actividad diaria realizada durante el periodo de práctica, de forma descriptiva e interpretativa. En este caso, la actividad registrada en las asesorías grupales y en los talleres investigativos.

Cuestionario social. Esta es una técnica de investigación en la cual se utilizan preguntas destinadas a un grupo de personas, donde la formulación de las preguntas es idéntica para todos los participantes. El cuestionario contiene una serie de preguntas que se contestan por escrito y estas tienen una formulación flexible. El tipo de pregunta que se utiliza en el cuestionario es la pregunta abierta.

Mapas conceptuales: Son instrumentos potentes para visualizar y analizar el grado de desarrollo de los conceptos aprendidos a través de la comprensión de textos, ya que los mapas son una evidencia que permite identificar las habilidades de pensamiento que posee un estudiante al momento de su diseño.

\section{Categorías deductivas}

Igualmente, desde el corte cualitativo, se plantean por parte de los investigadores, categorías deductivas de acuerdo con el análisis teórico y metodológico planteado. Las categorías deductivas consisten en las deducciones propias del investigador y que delimitan el rumbo de la investigación de acuerdo con la problemática planteada (Sandoval, 2002). Para este artículo, se describe la siguiente categoría deductiva:
Las categorías deductivas consisten en las deducciones propias del investigador y que delimitan el rumbo de la investigación de acuerdo con la problemática planteada (Sandoval, 2002). 


\begin{tabular}{|l|l|}
\hline Categoría deductiva & Operacionalización de la categoría deductiva \\
\hline $\begin{array}{l}\text { Contexto pedagógico frente a la } \\
\text { comprensión lectora }\end{array}$ & $\begin{array}{l}\text { Esta categoría muestra el análisis de los contextos } \\
\text { curriculares, didácticos y propios de los alumnos de } \\
\text { tercero de la Institución Educativa Sergio Camargo, } \\
\text { que inciden y circundan los procesos de la compren- } \\
\text { sión lectora }\end{array}$ \\
\hline
\end{tabular}

\section{Análisis de los resultados}

A través de la primera triangulación de la información, en cuanto al contexto de las formas de comprensión lectora de los educandos, se puede encontrar que una de las situaciones con relación a este proceso, es la forma como los educandos modelan la comprensión lectora de las indicaciones de los maestros.

El docente estimula todo tipo de situaciones pormedio de las indicaciones previas para la organización de la formas de lectura y comprensión de lectura en los niños, dando ejemplos y organizando los ejercicios, de manera tal que implícitamente hace de la lectura un deber y una obligación para los niños. Igualmente, este tipo de lectura basada en la copia de modelos tradicionales de la comprensión lectora, se caracteriza por el seguimiento de reglas asignadas previamente por el docente para cualquier ejercicio lector.

Así mismo, se encuentra que los docentes por medio del uso del mapa conceptual, dirigido como estrategia para mejorar la comprensión lectora, tienden de alguna forma a inducir a que los niños logren un aprendizaje significativo.

Según la triangulación de la información obtenida, se evidencia que cuando el docente "dicta" y ordena a los estudiantes la forma en que deben leer, genera una disminución en el interés propio y autónomo de leer y comprender por parte de los estudiantes, además que dificulta en los educandos la posibilidad de que ellos plasmen sus propias ideas. Dentro de esta misma categoría, se encuentra que paradójicamente el estilo comprensivo que prefieren los niños en la emulación de sistemas pedagógicos tradicionales, es la escogencia de un tipo de comprensión lectora basada en la memorización de los argumentos centrales de los textos.

Pormediodelas actividades desarrolladas en esta investigación, también se puede evidenciar que los estudiantes en su afán de copiar y comprender los textos rápidamente, tienden a buscar cómo repetir los contenidos de los documentos leídos. Es evidente que no conoce el significado de muchas de las palabras del texto, haciendo que se den errores de coherencia y cohesión en el momento de demostrar que han comprendido el texto.

Igualmente, se da otro fenómeno que afecta la comprensión lectora, es el nivel de control del docente sobre la lectura. Frente a este aspecto en esta categoría inductiva, se describe la relación que tiene la lectura de carácter modelado en vínculo con las acciones de control y regulación que desarrolla el maestro en las clases sobre la lectura sumado al afán del docente por mantener la disciplina 
cuando el estudiante se prepara para leer (Ospina, 2014). En primera medida, esta categoría inductiva destaca en cómo a través de los procesos de control de la lectura por parte del docente, el maestro fomenta diferentes formas de motivación en los estudiantes para comprensión lectora. Sin embargo, este tipo de condiciones de regulación afecta notablemente la comprensión lectora, porque el control ejercido por parte del docente limita y genera fronteras en la comprensión lectora del niño que, a su vez, fomenta la copia textual en la producción de escritos de los educandos.

Este tipo de dinámica recrea en el estudiante que la forma de comprender es una imposición y una obligación en los procesos académicos. Se puede encontrar que los estudiantes permanentemente solicitan al docente la explicación y definición de los diferentes vocabularios desconocidos emergentes, además que esta información dada por el docente es recopilada y representada escrituralmente de manera similar a lo dicho por el maestro. Sumado a lo anterior, también se puede encontrar que los estudiantes en estas solicitudes de explicación, necesitan descripciones detalladas del maestro de los esquemas de la comprensión textual que emplean y reproducen en la producción escritural. Es también visible cómo entre los propios compañeros solicitan y hacen expreso las demandas de explicación similares a las que le piden al docente.

En la parte investigativa de características cualitativas, se utiliza el sistema categorial o diseño sistemático, el cual permite la construcción y validación de categorías para la estructuración del análisis de los datos cualitativos. Por medio de este sistema de categorización, primero se realiza la codificación descriptiva y primer nivel de categorización, en esta fase se reúnen las unidades hermenéuticas correspondientes a cada una de las categorías de análisis o deductivas en la cual aparece un primer tipo de categorías descriptivas que emergen $\mathrm{o}$ surgen de un primer contacto con los datos recolectados. En segundo lugar, se realiza la codificación axial o relacional: estas unidades hermenéuticas ya clasificadas, se subdividen internamente dentro de cada categoría deductiva, haciendo un proceso de conceptualización de los datos obtenidos, vinculando las categorías dos o más observaciones descriptivas entre sí, a partir de este nivel de categorización surgen las denominadas categorías inductivas que son de orden más teórico y vinculan entre sí dos o más categorías descriptivas o teóricas de orden inferior (Sandoval, 1996, p. 159).

\section{Conclusiones}

La conclusión más importante de esta investigación es que la utilización del mapa conceptual como estrategia mejora el proceso de comprensión lectora; como instrumento dentro del aula, mejora en un alto porcentaje la interpretación, la producción oral y escrita de los estudiantes. En cuanto a la interpretación, se puede decir que: se presenta un avance significativo, ya que el uso del mapa conceptual permite un pensar y actuar flexiblemente con lo que el estudiante sabe y con los nuevos conocimientos, situaciones que le facilitan ir más allá de un pensamiento y acción memorística y rutinaria, facilitándole la construcción de su
En la parte investigativa de características cualitativas, se utiliza el sistema categorial o diseño sistemático, el cual permite la construcción y validación de categorías para la estructuración del análisis de los datos cualitativos. 
propio conocimiento, entendiendo por qué y para qué aprender.

El iniciar el trabajo con los mapas conceptuales en pro de mejorar la comprensión, admite que los estudiantes tengan una visión general no solo de lo que se espera de ellos, sino también que les brinda el espacio para proponer sus propias metas teniendo en cuenta sus conocimientos previos, favoreciendo la participación, autonomía y trabajo en equipo, ya que estos son consensuados entre todos. Es decir, que el proceso deja de ser unidireccional para convertirse en acciones participativas donde todos los miembros de la clase se tornan en agentes activos del proceso de enseñar y aprender.

El trabajo en equipo en la elaboración del mapa conceptual, favorece también el hacer interpretaciones adecuadas, ya que los estudiantes tienen la oportunidad de interactuar con sus compañeros, escuchar sus opiniones y contrastarlas, para llegar a una conclusión concertada y acertada.

\section{Referencias}

Ausibel, D. (1963). Teoría del aprendizaje significativo.

Bonilla, E. \& Rodríguez, P. (1997). Más allá del dilema de los métodos: las investigaciones sociales. Bogotá. Grupo editorial Norma.

Cañizalez Mesa, N. E., \& Benavides Rozo, F. A. (2018). La didáctica como herramienta de la reflexión docente. Educación Y Territorio, 6(11), 105 - 145. Recuperado a partir de https://jdc.edu.co/revistas/index.php/reyte/article/ view/48

Fals Borda, O. (2004). La Investigación Acción Participativa.

Kemmis, E. (1992). Cómo planificar investigación acción.

Mariño Díaz, L., Pulido Cortés, O., \& Morales Mora, L. (2016). Actitud filosófica, infancia y formación de maestros. Praxis \& Saber, 7(15), 81101. https://doi.org/10.19053/22160159.v7.n15.2016.5724

MEN. (2013). Plan Nacional de Escritura. Bogotá: Ministerio de Educación Nacional.Morata.

Miller, J. (1962). Teoría del procesamiento de información.

Sandoval, C. (2002). Programa de especialización en teoría, métodos y técnicas de investigación social, investigación cualitativa. Bogotá. Arfo Editores e Impresores Ltda.

Ospina Nieto, Y. (2014). Rescatar lo antropológico... una necesidad de la educación. Praxis \& Saber, 5(10), 193 - 218. https://doi.org/10.19053/22160159.3029

Torres, A. (1999). Estrategias y técnicas de investigación cualitativa. Facultad de ciencias sociales y humanas de UNAD. Santafé de Bogotá. Ediformas Ltda.

Vygotsky, L. (1991). Aportes del enfoque histórico cultural para la enseñanza. 